\title{
La infraestructura de soporte para la economía digital en Chile
}

\section{Rossella Cominetti C.}

Ex Jefa del Departamento de Regulación Económica,

Subsecretaría de

Telecomunicaciones (SUBTEL), Ministerio de Transportes y Telecomunicaciones de Chile rcominetti@indap.cl
Este artículo presenta un modelo que permite sistematizar temas asociados a las políticas de desarrollo de la infraestructura de soporte de la economía digital, identificando el papel del Estado y del sector privado. Asimismo, describe las principales iniciativas en materia de creación y mejoramiento de infraestructura y contenido en el caso de Chile, lo que permite apreciar el avance experimentado recientemente en el desarrollo de la economía digital en el país, así como los desafíos pendientes en este campo. Existe consenso en que Chile debe aproximarse en forma rápida y oportuna a aquellos países ya incorporados en la sociedad de la información. Este trabajo contribuye a identificar los factores más importantes para evaluar la política de acceso a la economía digital, uno de los pilares centrales que han de considerarse al diseñar políticas que permitan avanzar hacia la sociedad de la información. 


\section{I}

\section{Introducción}

El origen de un nuevo modelo en la industria de telecomunicaciones, a raíz de la digitalización de las señales y el desarrollo de Internet, ofrece las condiciones para una transformación radical en la forma en que las relaciones económicas y sociales pueden llevarse a cabo, en forma más eficiente y democrática, contribuyendo a una mayor competitividad, participación e igualdad. En particular, la sustitución de mecanismos tradicionales para realizar transacciones y trámites y para la comunicación en general por métodos que hacen uso intensivo de tecnologías de la información, está dando origen a lo que se ha denominado "la nueva economía digital", cuyos beneficios en materia de productividad hacen necesario incorporar rápida y masivamente a los agentes económicos en la red. Asimismo, las externalidades y oportunidades en materia de formación de capital humano asociadas al acceso y uso de las nuevas tecnologías plantean diversos desafíos a las políticas públicas.

Existe consenso en que una incorporación lenta al proceso de informatización no sólo significaría la pérdida de oportunidades ofrecidas por el acceso a la información -mercados más amplios, menores costos de producción, mayor capacitación - sino que también un posible deterioro de la posición alcanzada en la economía tradicional. En este sentido, la tecnología de la información se ha transformado en un factor determinante del crecimiento económico, y su desarrollo insuficiente puede incidir en un mayor ensanchamiento de las brechas con los países que se incorporen con más rapidez a la economía digital. A nivel nacional, el acceso masivo de la población a Internet puede ayudar a reducir las desigualdades que limitan el acceso equitativo a los beneficios del crecimiento económico.

Ante este nuevo fenómeno es preciso acelerar el ritmo de incorporación al acceso y uso de Internet como herramienta de información, transacciones, trámites y provisión de servicios sociales (por ejemplo, de educación y salud), y contribuir así a establecer un patrón de crecimiento sostenido y no excluyente que ayude a mejorar la calidad de vida de todos los ciudadanos. Para ello es indispensable la coordinación de los esfuerzos de los diversos actores involucrados, esto es, el Estado, las empresas, los proveedores de contenido, las universidades, las organizaciones no gubernamentales y la propia comunidad.
En el desarrollo de la infraestructura de la información, al Estado le corresponde principalmente establecer un sistema de incentivos para alcanzar niveles adecuados tanto en el acceso a las redes como en el contenido, para lo cual deberá:

- Promover el acceso universal a Internet.

- Eliminar barreras que interfieran en el acceso y uso de las redes.

- Establecer las condiciones necesarias para que haya inversión en la infraestructura de soporte para la economía digital y en el contenido.

- Velar por el desarrollo de recursos humanos compatibles con los requerimientos de la nueva economía digital.

- Convertirse en un agente catalizador del uso de Internet, mediante la modernización del aparato público.

- Coordinar las acciones públicas y privadas y las iniciativas de organizaciones no gubernamentales para asegurar un esfuerzo eficiente y aprovechar las sinergias entre ellas.

Chile fue el primer país latinoamericano que introdujo Internet: en enero de 1992 se efectuó la primera conexión, en el ámbito universitario. En 1998 -ante la percepción generalizada de que si bien el país había conseguido importantes logros en materia de telecomunicaciones, su desempeño en el ámbito de Internet era más débil que el de otros países de la región-, se formó una comisión presidencial orientada a identificar planes de acción para el avance de Internet en Chile. Con posterioridad, la administración que asumió en marzo de 2000 asignó una alta prioridad en la agenda de política pública al tema del acceso a las tecnologías de la información e Internet. El propio Presidente de la República, en su discurso anual ante el Parlamento, señaló en mayo de ese mismo año ${ }^{1}$ que "Chile debe asumir la vanguardia entre los países que usan las tecnologías de la información, especialmente Internet, como motor de un nuevo progreso. Un progreso que se basa en la flexibilidad de las empresas y no en su tamaño, en la inteligencia de la gente y no en la cercanía geográfica, en la cooperación y no en el

\footnotetext{
${ }^{1}$ Véase el discurso completo en http://www.presidencia.cl/cuenta/ index.htm .
} 
antagonismo". Para aplicar esta línea orientadora, y dándole continuidad a las comisiones creadas con anterioridad, se formó el Comité de Nuevas Tecnologías de Información y Comunicación. Integran este ente intergubernamental de carácter transversal autoridades de diversos ministerios y organismos públicos dotados de competencia en el ámbito de las nuevas tecnologías de la información y comunicación, y su misión es velar por el desarrollo de las nuevas tecnologías en Chile.

En este artículo se analiza la política asociada al desarrollo de la infraestructura necesaria para la incorporación de Chile y de todos sus ciudadanos a la sociedad de la información, y se evalúan los avances en esta materia. El documento se organiza en tres secciones, adicionales a esta introducción. La sección II presenta un modelo simple que permite conceptualizar las políticas para desarrollar la infraestructura de soporte de la economía digital, identificando los elementos básicos que han de ser considerados. La sección III examina y evalúa las principales medidas de política adoptadas recientemente por Chile en esta materia. Finalmente, la sección IV pasa revista a los principales desafíos que habrá que encarar para continuar con el desarrollo de la infraestructura para el acceso a la economía digital y contribuir así a posicionar al país entre aquellos incorporados a la sociedad de la información.

\section{II}

\section{La infraestructura de soporte de la economía digital}

Las nuevas tecnologías de información y comunicación (TIC) y el desarrollo de Internet constituyen piezas claves en la transformación que están experimentando las formas y mecanismos tradicionales de intercambio de información y que se ha extendido asimismo a la forma de intercambio de los bienes y servicios, dando origen a los términos "economía digital" o "nueva economía".

El concepto de economía digital dice relación principalmente con la sustitución de los mecanismos de intercambio. La forma de concretar las transacciones ha experimentado cambios sustanciales a lo largo del tiempo, permitiendo en cada caso reducciones significativas de los costos de transacción. Ha pasado del trueque al dinero, y del dinero, en sus diversas modalidades, al intercambio en el espacio virtual mediante dispositivos conectados a Internet, con la posibilidad de eludir los costos de transacción asociados a la intermediación. Cabe señalar que, en la nueva economía, la economía tradicional no desaparece: sólo se produce una modificación del mercado, donde demandantes y oferentes se encuentran en el espacio virtual para intercambiar bienes y servicios generados en la economía tradicional.

Dos son los requisitos básicos para el éxito de las transacciones a través de la red:

i) Infraestructura: infraestructura que permita el transporte de la información, haciendo posible el encuen- tro electrónico entre oferentes y demandantes; infraestructura de medios necesarios para que los acuerdos sean aceptados por las partes involucradas, e infraestructura para transportar los bienes transados electrónicamente desde el oferente hasta el demandante.

ii) Contenido: la información necesaria para que oferentes y demandantes conozcan adecuadamente los términos de la transacción.

La ausencia o limitaciones de uno de estos dos componentes amaga el éxito de las transacciones en la red. Por lo tanto, es preciso velar por el desarrollo armónico de ambos. ${ }^{2}$

El concepto de infraestructura de la información se ilustra en el gráfico 1, donde se aprecia que existe oferta de contenido de los sectores público y privado, y demanda de los bienes, servicios o información provistos por ambos sectores. Para que se concreten las

\footnotetext{
${ }^{2}$ Uno de los elementos centrales del diagnóstico elaborado en 1998 por la Comisión Presidencial de Nuevas Tecnologías de Información y Comunicación fue la identificación de un círculo vicioso entre ambos componentes. En efecto, se encontró que, por una parte, el escaso desarrollo de la infraestructura y el poco interés por operar a través de las redes tenía su origen en la escasez de contenidos $\mathrm{y}$, por otra, que la escasez de contenidos se debía a la baja penetración de Internet en los hogares y empresas. A raíz de ese diagnóstico se identificaron y llevaron a cabo proyectos emblemáticos que permitieran quebrar ese círculo vicioso, entre ellos, la incorporación del Estado en la red.
} 


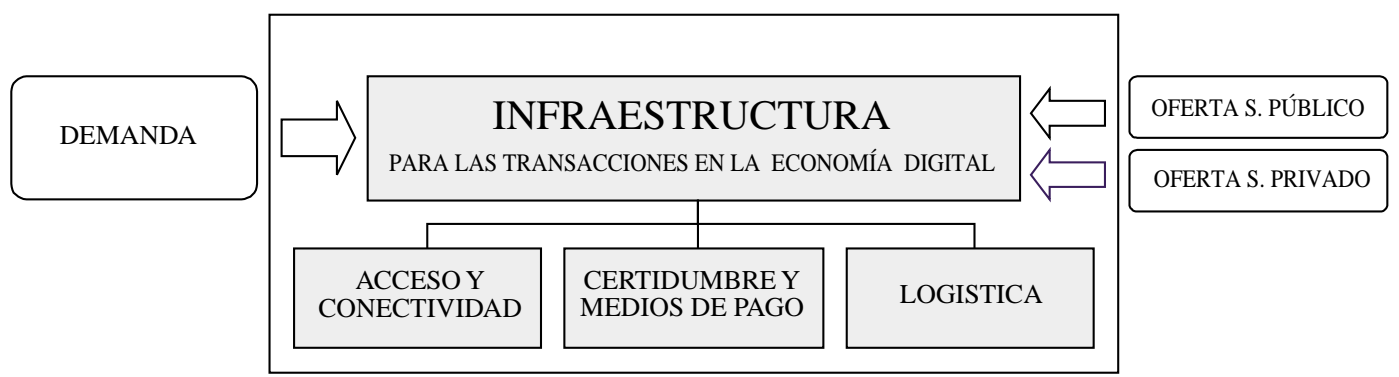

transacciones electrónicas se requiere una infraestructura de soporte que facilite la interacción entre oferentes y demandantes, haciendo posible las transacciones en la economía digital.

El concepto de infraestructura de soporte de la economía digital abarca el conjunto de elementos necesarios para garantizar el éxito de las transacciones a través de las redes de información. Los tres básicos son: la infraestructura de acceso, los medios de pago y de certidumbre, y la infraestructura de distribución. Cualquiera de los tres elementos anteriores que no opere adecuada y oportunamente, en un contexto de mercados globalizados y muy competitivos tras la incorporación de Internet, puede frustrar oportunidades de crear valor agregado a nivel nacional. Además, es esencial la presencia de una adecuada demanda y oferta de bienes y servicios en este mercado virtual, por lo cual es preciso aplicar una serie de medidas con el propósito de aprovechar los beneficios potenciales de las nuevas tecnologías.

El gráfico 1 permite ordenar las políticas de fomento del intercambio a través de medios digitales. En efecto, es posible identificar diversas medidas que involucran:

- Fortalecimiento de la demanda de bienes y servicios transados a través de la red.

- Desarrollo de infraestructura de acceso.

- Desarrollo de infraestructura de medios de pago y de certidumbre.

- Desarrollo de infraestructura logística.

- Fortalecimiento de la oferta de bienes y servicios transados en la red.

\section{Fortalecimiento de la demanda de bienes y servicios transados a través de la red}

En este ámbito se puede aplicar una variedad de medidas relacionadas con la formación de capacidades para el uso de Internet, lo que pasa no sólo por el dominio de los dispositivos conectados a Internet, sino también por reforzar el dominio del inglés, toda vez que en la actualidad alrededor de $78 \%$ de los sitios web y $96 \%$ de los sitios de e-commerce utilizan ese idioma. Asimismo, para hacer más atractivo el uso de Internet que el de métodos tradicionales para el intercambio de información, se pueden establecer diversos incentivos, como exenciones tributarias al comercio electrónico, ${ }^{3}$ o medidas para simplificar y hacer más rápidos los trámites. ${ }^{4}$

\section{Desarrollo de infraestructura de acceso}

La infraestructura de acceso está constituida por los medios de telecomunicaciones y dispositivos necesarios para acceder o conectarse a las redes y para transportar información que permita la comunicación electrónica bidireccional entre los actores que originan la información y los usuarios de ella.

Al ámbito de la infraestructura de acceso se refieren todas aquellas políticas encaminadas a incentivar el despliegue de dispositivos y redes de telecomunicaciones que permitan acceder a Internet y transportar datos, de manera no excluyente, eficiente y competitiva. Por lo tanto, en él se aplican las medidas de política y regulatorias que promueven la rápida absorción de las nuevas tecnologías y la competencia en el mercado de las telecomunicaciones, contribuyendo a abaratar el acceso a Internet, así como también las políticas relacionadas con la creación de infraestructura para los sectores vulnerables o aislados.

\footnotetext{
${ }^{3}$ Como las aplicadas recientemente en Estados Unidos a través de la ley HR 1054.

${ }^{4}$ Como se hizo en Chile y Brasil para las declaraciones de impuestos.
} 


\section{Desarrollo de infraestructura de certidumbre y seguridad}

Un elemento esencial en las transacciones es el dinero, esto es, el medio de pago que es aceptado por las partes involucradas. Requisitos básicos para tal aceptación son la seguridad del medio de pago empleado y la certificación de las transacciones. Asimismo, es indispensable la seguridad respecto de los agentes involucrados en una transacción, esto es, la identidad digital de los actores, así como la confianza en la inviolabilidad de los datos privados que se transportan a través de la red.

En este ámbito habrá que generar condiciones que garanticen la protección de la identidad digital, a través de la seguridad de la información que transita por las redes, que se cautela mediante sistemas de autenticación, certificación, privacidad y seguridad relativa a incidentes informáticos.

\section{Desarrollo de infraestructura logística}

Al ámbito de la infraestructura logística pertenecen aquellas políticas orientadas a garantizar un oportuno y eficiente despacho de los bienes o servicios adquiri- dos por vía electrónica. Entre las medidas que han de aplicarse se encuentran las relacionadas con un sistema logístico de back office que minimice los tiempos de despacho a través de un eficiente manejo de inventarios, y con la organización de la industria de distribución, a fin de asegurar la entrega oportuna e informada de las mercaderías al usuario.

\section{Fortalecimiento de la oferta de bienes y servicios}

Los beneficios potenciales del uso de Internet están estrechamente ligados a que los contenidos de la red sean de interés para los usuarios; por lo tanto, las políticas pertinentes deben estar orientadas a estimular la creación de tales contenidos. El sector público se puede transformar en un catalizador del uso de Internet, en la medida en que estimule el acceso a los servicios al público a través de Internet.

Al respecto, el principal desafío para dicho sector es el de trasladar el Estado al espacio virtual, de manera de acercar los servicios públicos a las personas. Y para el sector privado, el de desarrollar contenidos en la red, particularmente fortaleciendo a las pequeñas y medianas empresas, que tienen más dificultad para participar en un sistema de oferta virtual.

\section{III}

\section{Políticas para el desarrollo de la infraestructura de información en Chile}

Esta sección describe las principales políticas adoptadas en Chile para fomentar el desarrollo de la infraestructura de soporte de la economía digital, y evalúa la posición del país en cuanto a la infraestructura de acceso y conectividad.

La estrategia chilena ha sido de minimización de las regulaciones aplicables a la industria, de conexión a Internet y de estrecha colaboración público-privada en las diversas instancias de formulación de medidas para favorecer el desarrollo de Internet. Desde el comienzo, en la comisión presidencial para las nuevas tecnologías de información y comunicación se estableció el vínculo con el sector privado, como actor importante en la incorporación del país a la sociedad de la información. Posteriormente, en mayo de 2001 se creó la Alianza Público Privada; esta instancia, coor- dinada por el sector público ${ }^{5}$ e integrada por empresas del sector de las telecomunicaciones y de tecnologías de la información, del ámbito financiero, del transporte y de la industria de correos, se organizó para diseñar y establecer instrumentos y proyectos orientados a fomentar el desarrollo de la infraestructura tanto de acceso como de seguridad y de certidumbre y de distribución.

En esa misma dirección, el gobierno ha privilegiado la presencia del sector privado en el desarrollo de Internet, para lo cual promovió diversas misiones encabezadas por el Presidente de la República e integradas

\footnotetext{
${ }^{5}$ Específicamente, por la Unidad Especial de Infraestructura de Información creada en la SUBTEL. Véase www.chilefuturo.cl.
} 
por ministros de Estado y actores relevantes del sector privado, que apuntaban a establecer contacto entre el país y los puntos neurálgicos de la industria de las tecnologías e información; tales fueron, por ejemplo, las misiones a Silicon Valley y a Suecia en 2001. Entre los diversos resultados de esas misiones se hallan el establecimiento de la Fundación País Digital, iniciativa del sector privado; la puesta en marcha de un proyecto orientado a la creación de un parque tecnológico en la ciudad de Valparaíso y, sobre todo, el consenso acerca de la importancia del esfuerzo conjunto en el posicionamiento del país en el ámbito de las nuevas tecnologías.

A continuación se describen las principales políticas adoptadas en los diferentes ámbitos de la infraestructura de la información en Chile, ilustrándolas con programas específicos.

\section{Fortalecimiento de la demanda de bienes y servicios transados a través de la red}

Las políticas adoptadas en este campo se orientan principalmente a la capacitación de los recursos humanos para el uso eficaz de Internet. La demanda de infraestructura de la información está estrechamente ligada a la calidad y pertinencia de los contenidos disponibles en la red. En efecto, la experiencia de los telecentros comunitarios indica que un elemento central para su éxito y sustentabilidad es la creación de contenido local de interés para los habitantes del lugar, y que el grado de capacitación de los habitantes y su mayor o menor dominio del computador es un factor determinante del uso de tales telecentros. La capacitación mostró tener una incidencia directa en el aumento de la demanda de horas de navegación.

En lo que se refiere a la capacitación de los recursos humanos, se han puesto en práctica diversas iniciativas tendientes a reforzar la demanda de nuevas tecnologías. En el sector público se implantó un Programa de Formación en Tecnologías de Información para funcionarios públicos a lo largo de todo el país; se creó un Laboratorio de Transferencia de Tecnologías y se estudia la reconversión del programa de estudios de los institutos técnicos (que acogen el $40 \%$ de los estudiantes de enseñanza secundaria) para enfrentar los nuevos requerimientos de la infraestructura de la información.

Asimismo, a nivel de las pequeñas y medianas empresas, se creó el Programa de Capacitación en Internet para las Empresas (www.corfo.cl). Este programa busca que los empresarios vean en Internet una herramienta para mejorar la competitividad, ampliar los mercados y posibilitar nuevos negocios. Está diseñado para atender diferentes niveles de requerimientos de capacitación en Internet, adecuándose al grado de avance de las empresas en el proceso de absorción tecnológica.

\section{Desarrollo de infraestructura de acceso no excluyente}

Por infraestructura de acceso se entiende tanto el acceso de los usuarios a dispositivos que permitan establecer una comunicación, como las redes de acceso a la infraestructura básica de conectividad para la transmisión de datos.

\section{a) Acceso a dispositivos para el intercambio digital de información}

El acceso universal a las tecnologías de información es un objetivo de política prioritario para reducir la llamada brecha digital. Según información de noviembre de 2000 , ese año el $16.9 \%$ de los hogares chilenos tenía computador y el $8.3 \%$ estaba conectado a Internet. En términos de personas las cifras eran mejores: $29.6 \%$ y $15.9 \%$, respectivamente, lo que indica que hay acceso de las personas a computadores y a Internet en lugares diferentes a los hogares, así como a través de algunos programas comunitarios específicos.

La tasa relativamente baja de acceso de los hogares a Internet obedece en gran parte a que el principal medio utilizado es el computador personal, cuyo elevado costo constituye una importante barrera a la entrada. Si bien la evolución tecnológica permitirá introducir nuevos medios para acceder a Internet a mayor velocidad, como la televisión digital y los servicios móviles de tercera generación, es necesario fortalecer el acceso a terminales mediante programas de apoyo específicos.

Cabe señalar que a fines de 2001 el sector privado inició la instalación de teléfonos públicos especialmente habilitados para enviar $e$-mails y mensajes cortos a celulares, a precios accesibles para los usuarios, lo que contribuirá de manera significativa a la masificación de Internet. Asimismo, el mercado está generando soluciones que permiten acceder a Internet a través de infocentros.

El sector público, por su parte, ha puesto en vigor una serie de medidas orientadas a promover el acceso a computadores, las que pueden clasificarse según el carácter individual o comunitario del acceso. 
Entre las medidas aplicables para fomentar el acceso individual a dispositivos de conexión a Internet se encuentran los subsidios a la compra de equipos y las políticas crediticias focalizadas. Chile ha optado por la segunda alternativa, estableciendo un programa de créditos blandos para grupos de usuarios con intereses comunes, en este caso los profesores de colegios y las PYME. A diferencia de otros países, no ha avanzado en la implantación de programas específicos para masificar el acceso a Internet mediante la incorporación de equipos de menor costo.

La Corporación de Fomento de la Producción (CORFO) y el BancoEstado abrieron líneas de crédito para que las microempresas y las pequeñas empresas pudieran contar con equipos computacionales y con adiestramiento en Internet.

Para tales efectos, el BancoEstado ofrece dos líneas de crédito a las PYME (www.bancoestado.cl): un Crédito PC Fácil, para financiar la compra de un computador, dirigido al microempresario o pequeño empresario formalmente establecido y con al menos un año de funcionamiento; y un Crédito PC Internet, destinado a financiar la compra de equipos de computación para todo tipo de establecimientos educacionales, tanto particulares subvencionados como municipalizados, permitiéndose habilitar salas de computación y equipos computacionales para ejecutar tareas administrativas del establecimiento.

Asimismo, en 2001 la CORFO puso en marcha un programa de provisión de 10000 conjuntos informáticos (equipos y servicios) destinados a pequeños empresarios y profesores, con financiamiento preferencial. Este programa se repitió a fines de ese año por el mismo número de soluciones informáticas.

La política de acceso comunitario a los servicios de telecomunicaciones ha estado orientada principalmente a fortalecer la infraestructura para los sectores vulnerables o aislados, a través del subsidio a la inversión. Merced a la política de acceso universal implantada en Chile desde 1995 se ha logrado extender la infraestructura básica de telecomunicaciones —redes alámbricas, inalámbricas y satelital— a lo largo del país, particularmente en las zonas rurales, por lo cual su cobertura es amplia, ${ }^{6}$ y se espera extenderla a infraestructura que permita el acceso universal a Internet.

\footnotetext{
${ }^{6}$ El programa Fondo de Desarrollo de las Telecomunicaciones, puesto en marcha en 1995, permitió elevar, en el lapso de cinco años, la cobertura de teléfonos públicos en casi todo el país. Solamente el $3.2 \%$ de los hogares aún carece de acceso a telefonía, vale decir, ni siquiera accede a teléfonos públicos.
}

Hay una serie de iniciativas para la promoción del acceso comunitario a las tecnologías de la información que ya han sido puestas en marcha.

Red Enlaces. En el contexto del programa de reforma educacional, en 1993 se dio inicio a un proyecto experimental de informática y redes digitales para las escuelas subvencionadas de Chile, conocido como Red Enlaces, cuyo propósito es integrar las tecnologías de información en el sistema educacional como recurso de enseñanza y aprendizaje, capacitar a los profesores y desarrollar contenidos pertinentes. $\mathrm{Al}$ año 2000 el $90 \%$ de los estudiantes de Chile contaba en su escuela con una sala de computación conectada a Internet (100\% de cobertura de la educación media y $50 \%$ de la básica). En el año 2001 se inauguró un portal educativo, www.educarchile.cl, que permitió a la comunidad un importante avance en materia de contenido educativo relevante para la formación de capital humano.

Programa Nacional de Infocentros Comunitarios. Este programa es una instancia de coordinación y generación de alianzas interinstitucionales. Opera a través de una mesa de trabajo gubernamental, que está orientada a la coordinación de las acciones públicas en esta materia, a la creación de alianzas y al diseño de políticas que promuevan el acceso universal comunitario, en estrecha coordinación con las iniciativas no gubernamentales. Se ha hecho un catastro nacional de infocentros (www.gobiernodechile.cl y www.mapas.moptt.cl), que registra actualmente 184 de ellos; este número incluye 18 infocentros para las PYME, que se elevarán próximamente a 60 . Asimismo, durante el año 2002 se proyecta crear alrededor de 366 infocentros en instalaciones que la Dirección de Bibliotecas, Archivos y Museos tiene en todas las comunas del país: la iniciativa está a cargo de dicha institución y de la Fundación Gates. Entre las medidas de despliegue de infocentros a lo largo del país destacan los proyectos de reciclaje de computadores y de telecentros comunitarios.

Proyecto de Reciclaje de Computadores para Infocentros. Esta iniciativa, originada en 2001 en el ámbito de la Alianza Público Privada, apunta a la creación de infocentros administrados por organismos no gubernamentales y organizaciones comunitarias, con equipamiento y servicios aportados por empresas privadas. El papel del Estado es principalmente de gestión, focalización y selección de las instituciones a cargo de la administración de los infocentros, así como de coordinación entre las empresas y las organizaciones de la sociedad civil que los gestionan. A fines de 2001 se había puesto en marcha 10 infocentros de esta 
naturaleza, y se proyectaba llegar a 100 con soluciones recicladas a fines de 2002.

Telecentros Comunitarios. Este programa, asociado al Fondo de Desarrollo de las Telecomunicaciones (www.subtel.cl), pretende dar a las comunidades locales una solución comunitaria integral en materia de servicios de telecomunicaciones, entre los que se encuentra Internet. La puesta en marcha de este programa de fondos de subsidio concursables para la instalación de telecentros comunitarios busca generar infraestructura comunitaria de telecomunicaciones e Internet en localidades aisladas. El financiamiento de este subsidio proviene de los ingresos generales de la nación, de manera que no tiene efecto distorsionador en la industria. Asimismo, su asignación es eficiente, por cuanto se concursan aquellos proyectos con mayor rentabilidad social y se otorga el subsidio a los postulantes que, cumpliendo con los requisitos básicos, soliciten el monto menor. Actualmente funcionan con éxito cinco telecentros comunitarios, en calidad de proyecto piloto, lo que permitirá su despliegue durante el año 2002.

\section{b) La infraestructura básica de conectividad para el acceso a la economía digital}

La política de telecomunicaciones chilena ha estimulado un acelerado desarrollo de la infraestructura de telecomunicaciones, la que exhibe un estándar elevado frente a otros países con similar ingreso per cápita. Debido a la temprana liberalización del mercado, ${ }^{7}$ a la privatización de las principales compañías de telefonía fija y de larga distancia a fines de los ochenta, y a la aplicación de un marco regulatorio que fomenta la inversión, la competitividad del mercado y el acceso universal, la inversión en telecomunicaciones ha sido creciente, con lo cual ha aumentado el peso del sector en la economía nacional.

El acceso a Internet en Chile se ha ampliado fuertemente desde mediados de 1999, debido a la baja del precio de la conexión conmutada, al progreso tecnológico que ha permitido el acceso a Internet a mayor velocidad — con precios que los usuarios residenciales y las PYME puedan pagar-, y a un marco de regulaciones mínimas en un mercado que opera de manera competitiva. ${ }^{8}$ Actualmente, la tasa de penetración del servicio de acceso a Internet supera el $20 \%$, merced al

\footnotetext{
${ }^{7}$ A comienzos de la década de 1980.

${ }^{8}$ A nivel de hogares, la infraestructura de acceso a Internet a noviembre de 2000 señalaba que el 17\% de los hogares tenía computador y el $8.2 \%$ de los hogares poseía conexión a Internet.
}

gran incremento de las conexiones causado por la irrupción de nuevas modalidades de conexión de banda ancha a partir de 2001.

Si bien la infraestructura básica de conectividad, entendida como la infraestructura básica de acceso a Internet, que está conformada principalmente por la red pública de telefonía fija, muestra un desempeño satisfactorio a nivel regional, el país todavía exhibe un déficit considerable en comparación con los países desarrollados (cuadro 1).

Respecto de la organización industrial, aunque ha habido avances sustantivos en cuanto a la presencia de nuevos operadores y el desarrollo de nuevos servicios, la estructura de mercado del sector muestra aún importantes grados de concentración: particularmente en lo que respecta al acceso a la "última milla", el operador dominante todavía concentra alrededor de $80 \%$ del mercado. En otros servicios, como los de telefonía móvil, larga distancia e Internet, si bien los operadores son muchos (4, 13 y 42 , respectivamente), el mercado tiende a concentrarse fuertemente en dos o tres de ellos, los que a su vez se encuentran integrados verticalmente en todos los negocios.

El acceso a la economía digital requiere infraestructura de acceso y conectividad en tres niveles:

i) Conectividad internacional. Para alcanzar la plena integración del país a los sistemas de comunicación globales se necesita infraestructura que permita la conexión con sistemas localizados fuera del país, mediante enlace internacional, y, por ende, con la red

\begin{tabular}{|c|c|c|c|}
\hline \multirow{2}{*}{ ADRO 1} & \multicolumn{3}{|c|}{$\begin{array}{l}\text { Comparación internacional de la } \\
\text { cobertura de los servicios de } \\
\text { telecomunicaciones, } 2000 \\
\text { (Líneas telefónicas, abonados y usuarios } \\
\text { por cada } 100 \text { habitantes) }\end{array}$} \\
\hline & $\begin{array}{c}\text { Telefonía } \\
\text { fija }\end{array}$ & $\begin{array}{c}\text { Telefonía } \\
\text { móvil }\end{array}$ & $\begin{array}{c}\text { Usuarios } \\
\text { de Internet }\end{array}$ \\
\hline Argentina & 21.3 & 16.3 & 6.8 \\
\hline Brasil & 18.2 & 13.6 & 2.9 \\
\hline Colombia & 16.9 & 5.3 & 2.1 \\
\hline Chile & 22.1 & 22.2 & 16.6 \\
\hline México & 12.5 & 14.2 & 2.7 \\
\hline Perú & 6.4 & 4.8 & 9.7 \\
\hline Uruguay & 27.8 & 13.2 & 11.1 \\
\hline Venezuela & 10.8 & 21.7 & 3.9 \\
\hline Estados Unido & 70.0 & 39.8 & 34.7 \\
\hline España & 42.1 & 60.9 & 13.3 \\
\hline Finlandia & 55.0 & 72.0 & 37.2 \\
\hline Inglaterra & 58.8 & 72.7 & 25.8 \\
\hline Italia & 47.4 & 73.7 & 23.0 \\
\hline Suecia & 68.2 & 71.7 & 45.6 \\
\hline
\end{tabular}

Fuente: Unión Internacional de Telecomunicaciones (UIT) y SUBTEL. 
global de redes y, de esa forma, poner al alcance de las personas y empresas del país las oportunidades existentes en cualquier parte del mundo.

A comienzos de 2001 entraron en operación dos anillos de cable submarino de alcance continental que suministran capacidad internacional de hasta 2.56 terabits por segundo, conectando la red nacional con los sistemas que operan en los Estados Unidos y, a través de éstos, con el resto del mundo. Esto significó un salto cuantitativo en materia de capacidad de conectividad internacional y eliminó una importante barrera al desarrollo de las tecnologías de la información en el país.

ii) Conectividad nacional (backbone). Es necesario disponer de redes para la conexión entre comunidades en los ámbitos local y nacional, a velocidades que permitan satisfacer las exigencias del desarrollo económico y social del país; esas redes constituyen la columna vertebral del sistema.

En materia de conectividad nacional, la infraestructura de acceso a Internet puede ser vista como una red troncal y múltiples redes de acceso, siendo hoy la red pública telefónica la más utilizada. La red troncal está constituida fundamentalmente por redes de fibra óptica que proporcionan la conectividad en el ámbito nacional y también en el internacional, y que permiten atender los requerimientos de capacidad sin restricciones.

En Chile, la zona que se extiende desde Arica a Puerto Montt cuenta con tres redes de fibra óptica a nivel nacional, aparte de redes locales en las principales ciudades del país. En consecuencia, existe una oferta de servicios suficiente y competitiva en la mayor parte del país, aunque permanece desatendida la zona más austral, donde se estudia la participación subsidiaria del Estado.

iii) Conectividad local o acceso al usuario final. Es preciso disponer de medios de transmisión a través de los cuales se pueda llegar a nodos digitales - principalmente conmutadores (switches) o enrutadores (routers) capaces de dirigir sus comunicaciones digitales hacia usuarios o contenidos ubicados dentro y fuera del país.

En lo que respecta a la conectividad local, es decir, a las redes de acceso a Internet que realizan la conexión entre los usuarios y el sistema de conectividad nacional, la red telefónica pública es la que hace actualmente la mayor contribución. ${ }^{9}$ Más del 53\% de

\footnotetext{
${ }^{9}$ En Chile, el $100 \%$ de las redes de conmutación y transmisión instaladas para la telefonía local están digitalizadas desde 1993, lo que ha permitido al país contar desde los primeros años de la década de 1990 con una adecuada infraestructura para la transmisión de datos.
}

los hogares en Chile cuenta con servicio telefónico en el hogar, mayoritariamente en las áreas urbanas y sectores de más altos ingresos. Una gran proporción de esa red utiliza la tecnología tradicional de par de cobre; el resto emplea tecnologías apoyadas en redes de TV cable y tecnologías inalámbricas (wLL —wireless local loop-y satelital en áreas rurales).

Un elemento central de la política de telecomunicaciones en los últimos años ha sido el fomento de la competencia en el mercado de acceso a la "última milla". Con el advenimiento de las señales digitalizadas, las diferencias tecnológicas entre redes se han comenzado a tornar irrelevantes, fenómeno que se conoce como convergencia. Las tecnologías de redes están incorporando el protocolo IP como estándar de soporte de casi todos los servicios conocidos y en desarrollo, poniendo éstos al alcance de las personas y las empresas, cualquiera sea el operador y el tipo de red. De esta forma, la convergencia de medios ofrece una oportunidad para profundizar la competencia como condición necesaria para el adecuado desarrollo de una infraestructura de redes de telecomunicaciones que ofrezcan más y mejores alternativas de conexión local a los usuarios.

A nivel nacional, se espera la introducción de nuevas tecnologías que contribuirán al acceso de banda ancha y a promover la competencia en el mercado de acceso al usuario final. Recientemente se ha iniciado la etapa experimental para la incorporación de la tecnología PLC (Power Line Communications), que permite establecer una red de acceso al backbone de Internet a través de las líneas eléctricas que suministran energía domiciliaria. Por otra parte, mientras se espera la introducción del servicio de tercera generación $(3 \mathrm{G})$, la industria de telefonía móvil ha incorporado tecnologías que permiten transmitir datos a mediana velocidad, las que se denominan genéricamente 2.5G. Por último, en materia de televisión digital el país enfrenta desafíos asociados a la definición del estándar técnico de televisión digital terrestre y al diseño del modelo de transición desde la televisión analógica: hay que garantizar el acceso no excluyente a esta tecnología y la generación de condiciones que permitan a los canales de televisión posicionarse en el sector de las telecomunicaciones de manera competitiva, en un contexto de convergencia. ${ }^{10}$

Al sector privado le corresponde introducir las nuevas tecnologías, ya sea en las redes existentes o

10 Véase subTel (2000). 
creando nuevas redes en forma oportuna y eficiente, mientras que al Estado le compete perfeccionar y adaptar la normativa para procurar la oportuna incorporación de nuevas tecnologías, así como simplificar la tramitación de las autorizaciones y vigilar el comportamiento de los mercados para asegurar una sana competencia.

\section{Desarrollo de infraestructura de seguridad y de certidumbre}

Un factor clave para el desarrollo de la economía digital es la existencia de medios de pago reconocidos, seguros y de bajo costo. La naturaleza del comercio electrónico, que permite que oferente y consumidor estén ubicados en países y sistemas legales distintos, hace necesario disponer de medios de pago conocidos y aceptados. Para ello se requiere un marco de normas de amplia aceptación y una adecuada institucionalidad que garantice la eficiencia de los procedimientos de certificación, que pueda arbitrar las transacciones electrónicas y la información comercial, y que proteja de abusos y engaños a las partes.

Las deficiencias en las condiciones de confiabilidad, entendiendo por ella tanto la seguridad de las redes, medios de pago y procedimientos de certificación como la garantía de privacidad, pueden ser una barrera al comercio electrónico —en particular a la prestación de servicios a través de las redes-y poner en peligro la competitividad de la economía digital, elevando el riesgo del país en materia de operaciones electrónicas.

La valoración y uso que las personas hacen de las redes de información están condicionados por el grado de certidumbre que éstas puedan ofrecer. ${ }^{11}$ En otras palabras, el uso de la red es altamente sensible a la percepción de los usuarios de que existen condiciones que garantizan la seguridad y privacidad de la información que transita a través de las redes. Así, una política que contribuya a fortalecer la percepción de seguridad de las redes, de privacidad, integridad y autenticidad de las comunicaciones e información, así como de certidumbre en los sistemas de transferencia de valor, estimula la creación de valor agregado a través de la red.

En el país han surgido dos entidades que brindan servicios de certificación, que pueden convertirse en

\footnotetext{
${ }^{11}$ Un informe en Estados Unidos señala que en 2001 el $42 \%$ de las denuncias de fraude examinadas se referían a casos de robos de tarjetas de crédito o datos personales a través de Internet.
}

un instrumento básico para generar confianza en intercambios del tipo empresa a consumidor conocidos como B2C (business to consumers). Y recientemente se aprobó la firma digital, lo que contribuirá a la superación de restricciones legales que limitan el intercambio electrónico.

En materia de medios de pago, se ha avanzado en la habilitación del sistema tradicional de tarjetas de pago para transacciones monetarias a nivel nacional a través de la red. No obstante, para masificar los sistemas de transferencia de valor y acelerar el avance en la política de acceso a la economía digital, es preciso abordar el tema de la generación de medios para transferir valor que puedan ser utilizados por los sectores de menores ingresos, los cuales no acceden a los instrumentos financieros tradicionales. Un desafío importante en materia de medios de pago es el de enfrentar su elevada concentración en Transbank, empresa que administra todas las tarjetas de crédito financieras tradicionales. A este respecto, cabe resaltar que las grandes tiendas comerciales han conseguido competir de manera significativa en el mercado del crédito a plazo a través de tarjetas comerciales.

En cuanto a incidentes informáticos, es preciso fortalecer la disponibilidad de dispositivos inteligentes destinados a reducir la posibilidad de ataques que pongan en riesgo la integridad de la información y la privacidad de las personas. Asimismo, es indispensable formar equipos altamente calificados para actuar de manera dinámica ante las distintas formas que puedan adoptar las amenazas a la seguridad en el intercambio de información.

Entre las principales iniciativas adoptadas por el sector privado y sobre todo por el sector público respecto a infraestructura de seguridad y de certidumbre en Chile, se encuentran las que se enumeran a continuación.

Privacidad. Se puso en marcha el proyecto Confiare, impulsado por la Cámara Nacional de Comercio y un conjunto de empresas y asociaciones relacionadas con las tecnologías de la información, en el cual participan también instituciones del sector público. Esta iniciativa sin fines de lucro apunta a construir confianza entre usuarios y proveedores de Internet respecto al uso de la información, mediante la certificación de los sitios afiliados que cumplen con ciertos estándares de políticas mínimas de privacidad.

Informativos básicos de seguridad en Internet para usuarios y recomendaciones sobre prácticas de seguridad. En el ámbito de la educación a los usuarios, se elaboraron informativos de seguridad destina- 
dos a diferentes tipos de usuarios de Internet (como los usuarios residenciales, las PYME), y otro para padres, niños y adolescentes internautas. En ellos se señalan, en forma didáctica, algunas medidas básicas de seguridad en el uso de Internet. Además, se preparó un informe destinado a identificar y formular un conjunto de recomendaciones operativas en temas de seguridad informática. Los informativos de seguridad se encuentran en el sitio web de la Subsecretaría de Telecomunicaciones, así como en los portales de los principales proveedores de acceso a Internet (ISP) del país.

Ley de firma electrónica. Desde 1999 se encuentra vigente la firma electrónica en el sector público. La reciente aprobación de la ley de firma digital en enero de 2002, que equipara el documento impreso con el electrónico y reconoce la validez y valor probatorio de los actos y contratos celebrados por medios electrónicos, contribuirá a dar confianza y a reafirmar la seguridad tecnológica y jurídica de las transacciones electrónicas por Internet. A mediados de 2002 se contará con un reglamento que permitirá poner en marcha el sistema de acreditación y certificación para las empresas privadas, los organismos de gobierno y los poderes judicial y legislativo del Estado.

Desarrollo de políticas de seguridad de la información dentro del sector público. Esta iniciativa interministerial está dirigida a establecer la política general de seguridad de la información dentro de la administración pública y las herramientas metodológicas para su implementación, así como el control de gestión de los riesgos informáticos y los mecanismos de auditoría. Se creó una red de cooperación contra el cibercrimen y se encuentran en elaboración procedimientos y acuerdos que involucran a la Policía de Investigaciones, el Ministerio de Justicia, la Subsecretaría de Telecomunicaciones, los proveedores de acceso a Internet y las empresas de seguridad, con miras a coordinar la denuncia, investigación y sanción de los delitos informáticos.

\section{Desarrollo de infraestructura logística}

La existencia de mecanismos de distribución eficientes es un requisito para el comercio electrónico. La distribución no sólo involucra la entrega oportuna de los bienes adquiridos electrónicamente, sino también la existencia de un sistema logístico que garantice la disponibilidad de los bienes que están siendo adquiridos y su oportuno despacho.

Para que exista una industria de distribución eficiente hay que generar condiciones para que la oferta de servicios sea de calidad, vale decir, que el servicio de distribución sea oportuno, que las mercancías sean recibidas en buen estado, que se pueda rastrear el producto transportado, entre otros atributos. Asimismo, es deseable una diversidad de alternativas de servicios disponibles para distintas combinaciones de precio y calidad, que permita a los usuarios escoger la combinación que mejor se adecue a sus necesidades y restricciones. Para ello es necesario estimular la competencia y la transparencia del mercado del servicio de distribución.

La industria de correos y encomiendas debe ajustarse a las necesidades del comercio electrónico, lo que exige una revisión de los procedimientos de despacho y la readecuación de los sistemas e infraestructura de transporte, almacenamiento y distribución, y de los sistemas de información en línea. La industria de correos en Chile ha experimentado importantes progresos, con el desarrollo de nuevos servicios, para adecuar la industria a los requerimientos del comercio electrónico. Por una parte, empresas privadas ya instaladas, como Lan Courier ( $w w w$.lancourier.com), han estado adaptándose a tales exigencias, ofreciendo nuevos servicios en el ámbito de la distribución y la logística (por ejemplo, rastreo en línea y distribución justo a tiempo) y aportando transparencia y menores costos. Por otra parte, han surgido nuevas empresas de distribución que aspiran a alcanzar estándares de calidad internacionales y que como ChilePost (www.chilepost.cl), junto a otros operadores de carácter internacional, contribuyen al desarrollo de mercados más competitivos.

En el sector público, la Empresa Correos de Chile enfrenta importantes desafíos de modernización y de adecuación al comercio electrónico, toda vez que debe competir con operadores de elevados estándares de calidad, y aún exhibe debilidades en materia de simplicidad de trámites y orientación del servicio al cliente en el ámbito del comercio electrónico. En aquellos países en que la principal empresa de correos todavía es estatal, ella desempeña un importante papel: debido a su presencia a lo largo del país, constituye la única alternativa de distribución en gran parte del territorio nacional, lo que es fundamental para la masificación del comercio electrónico. Por otra parte, su amplia infraestructura abre la posibilidad de instalar soluciones de acceso masivo a Internet, particularmente en las zonas más apartadas.

Por último, para ajustar la logística a las exigencias de las nuevas tecnologías, es preciso revisar los instrumentos destinados a promover la incorporación de nuevas prácticas de gestión basadas en tecnologías 
de la información, particularmente en lo que se refiere al back office de las pequeñas y medianas empresas.

\section{Fortalecimiento de la oferta de bienes y servi- cios}

La política de fortalecimiento de la oferta de bienes y servicios ha estado asociada principalmente a la creación de contenidos del sector público, con el propósito de generar un efecto catalizador que incentive el uso de Internet por la población. Pero el sector privado también ha hecho un esfuerzo significativo y últimamente ha mostrado mucho dinamismo. En efecto, entre marzo de 2000 y marzo de 2001, el número de tiendas virtuales aumentó de 57 a 481 y el número de productos del tipo B2C pasó de 37000 a $340000,{ }^{12}$ en tanto que el volumen transado ${ }^{13}$ se duplicó.

En lo que toca al contenido, el sector público ha exhibido un gran dinamismo, como resultado de una decisión de avanzar hacia el gobierno electrónico mediante la puesta en red de diversos servicios públicos. Emblemático es el caso del Servicio de Impuestos Internos (SII), que desde el año 1999 ofrece la oportunidad de efectuar declaraciones de impuestos vía Internet, lo que le ha permitido ocupar una posición de liderazgo a nivel internacional. En el ejercicio tributario de 2001, el número de declaraciones de impuestos por Internet alcanzó a 788 645, lo que corresponde al $41.6 \%$ de los contribuyentes. De manera similar, actualmente es posible efectuar determinados trámites por Internet ante algunos servicios públicos, ahorrando tiempo y papeleo: entre otros, el pago previsional en línea al Instituto de Normalización Previsional (www.inp.cl) y la obtención de certificados varios en el Registro Civil (www.registrocivil.cl). Asimismo, actualmente se encuentran disponibles o en preparación variados portales tendientes a acercar el Estado a la ciudadanía, algunos de los cuales se describen brevemente a continuación.

a) Sistema de información de compras y contrataciones del sector público (compraschile.cl)

Este sistema permite conocer los bienes y servicios que el Estado chileno requiere y fue diseñado para distribuir y mantener actualizada a través de Internet la información sobre sus operaciones de abastecimiento,

\footnotetext{
12 Véase Cámara de Comercio de Santiago (2001).

13 En 2001, el comercio del tipo B2C alcanzó a 65 millones de dólares y el de empresa a empresa, conocido como B2B (business to business), a 900 millones de dólares.
}

quedando cada procedimiento debidamente documentado en línea de manera transparente. Las instituciones del Estado publican en él sus requerimientos de bienes y servicios, de modo que cualquier usuario pueda conocerlos y, así, transformarse en un potencial oferente.

\section{b) Ventanilla única}

Este proyecto busca que el ciudadano que necesita algún servicio suministrado por el sector público pueda acudir a un solo lugar, y que las instituciones involucradas y los trámites en cada una de ellas resulten transparentes para el usuario. El objetivo de la ventanilla única es crear un acceso menos costoso a los servicios del Estado, de manera de mejorar la eficiencia y la comodidad de los trámites ante el Estado a fin de satisfacer las demandas de los ciudadanos y permitirles cumplir sus obligaciones o solicitar autorizaciones, reduciendo el tiempo y los costos de los procesos tanto para el Estado como para los usuarios.

En el contexto del proceso de modernización del Estado se creó el sitio $w w w . t r a m i t e f a c i l . g o v . c l$, en el que se encuentra información sobre los distintos trámites que las personas deben hacer ante las distintas reparticiones públicas, permitiéndoles efectuar directamente, en línea, aquél que necesitan. Este sitio constituye una ventanilla única para la atención (en línea) de las solicitudes de los ciudadanos: en la actualidad es posible realizar en él 38 trámites y obtener información acerca de algunos 700 .

También se puso en marcha una ventanilla única para la atención en línea de las empresas, denominada www.sitioempresa.cl, que permite acceder a diferentes sitios para realizar trámites. Contiene un sitio especialmente diseñado para quienes desean formar una empresa y necesitan saber cuáles son los procedimientos y trámites con que deben cumplir para poder estructurarla e ingresar al mercado.

\section{c) Uso de las tecnologías de información dentro del sector estatal}

Este proyecto apunta a introducir el uso, dentro de las instituciones públicas y entre ellas, de las nuevas tecnologías de información y comunicación, para elevar la eficiencia y eficacia de su gestión mediante el ahorro del tiempo y los recursos asociados a su demanda de documentación. Para ello, el proyecto contempla identificar los procedimientos internos recurrentes de los distintos organismos y rediseñarlos considerando el uso de esas nuevas tecnologías.

Asimismo, busca ampliar el uso de las tecnologías de la información en el sector estatal más allá del correo electrónico y las páginas web, y emplearlas para 
apoyar la gestión interna, contando con un soporte técnico común para las instituciones de menor tamaño que permita aprovechar economías de escala, y estableciendo mecanismos de comunicación más expeditos mediante un sistema de identificación electrónica común como gobierno central.

\section{d) Infraestructura para los servicios públicos}

Se está estudiando incorporar un sistema de concesión de infraestructura digital, a través de la cual el Estado pueda encomendar a un agente privado la construcción de infraestructura física para cobijar su plataforma electrónica de servicios públicos, entregando al sector privado tanto la gestión de la información asociada a ese bien público, como la distribución de los productos requeridos por los particulares. e) Otras iniciativas para mejorar el contenido de Internet dentro del sector público

Existen numerosas iniciativas de las diferentes instituciones públicas que surgen de manera dinámica, contribuyendo a mejorar el contenido de Internet y a dar mayor eficiencia y transparencia a los procesos de tramitación de servicios. Entre ellas se hallan la tramitación en línea de las concesiones de telecomunicaciones, implantada a fines de 2001 por la Subsecretaría de Telecomunicaciones, y la decisión del Ministerio de Educación de que a partir de 2002 las postulaciones de los estudiantes universitarios al crédito fiscal sólo se harán a través de Internet, para lo cual creó 300 infocentros con el equipamiento necesario para asegurar el acceso de todos los postulantes a este mecanismo.

\section{IV}

\section{Desafíos de política para la infraestructura de la información}

La infraestructura de la información ha mostrado avances sustantivos en los años recientes, como resultado de una decisiva opción de llevar al país hacia la sociedad de la información. En este proceso han sido claves la voluntad y el esfuerzo tanto del sector público como del sector privado. No obstante, el país se encuentra aún en pleno proceso de construcción de la infraestructura de soporte de la economía digital. Por lo tanto, persisten numerosos desafíos que deberán ser enfrentados de manera conjunta por ambos sectores en los próximos años, para permitir la masificación del uso de Internet como herramienta de trabajo, comercio, ocio, educación e integración de los ciudadanos.

\section{Desarrollo eficiente de la infraestructura de telecomunicaciones}

En el contexto de la globalización, el crecimiento sostenido y sustentable de la economía estará asociado cada vez más al desarrollo de la infraestructura de la información, que se viene a sumar a los factores productivos fundamentales de una estrategia de crecimiento con equidad.

El desarrollo eficiente de la infraestructura de telecomunicaciones, a su vez, se vincula con la exis- tencia de mercados competitivos. En situación de convergencia de medios, en que un mismo servicio puede ser transportado a través de redes de distinta tecnología y en que a través de distintas redes es posible transportar los mismos servicios, se precisa garantizar al menos la competencia entre redes.

Desde comienzos del decenio de 1980 y con mayor énfasis a partir de la década de 1990, la política de telecomunicaciones en Chile se ha orientado a profundizar la competencia como condición necesaria para lograr una adecuada infraestructura de redes de telecomunicaciones, que es básica para el acceso a la sociedad de la información. En este sentido, hay que profundizar los mecanismos de mercado, lograr que la infraestructura y la calidad de los servicios se extienda a sectores de bajos ingresos aún desatendidos, ${ }^{14} \mathrm{y}$ avanzar en la generación de condiciones para incorporar nuevas tecnologías en la "última milla" que permitan transitar hacia la desregulación de este segmento. La intervención pública se reservaría exclusivamente para la regulación técnica, la corrección de distorsiones

\footnotetext{
${ }^{14}$ Esto significa facilitar la aplicación de mecanismos como los sistemas de prepago y otros que han permitido la rápida masificación de la telefonía móvil.
} 
y la aplicación de políticas de carácter subsidiario. El mismo principio es extensible al resto de los segmentos de la industria, donde el desarrollo de la tecnología y los servicios obligan a asegurar la oportuna asignación del espectro radioeléctrico y a establecer regulaciones compatibles con el contexto de convergencia.

El perfeccionamiento y la ampliación de los mecanismos para reducir las prácticas anticompetitivas, así como la eliminación de las barreras a la entrada de empresas y a la salida de usuarios, contribuyen a promover la competencia dentro de una misma red. Entre tales mecanismos se encuentran la desagregación de redes, la portabilidad del número telefónico y la fiscalización y sanción rigurosas de las conductas anticompetitivas. También la regulación tarifaria y una política de atribución del espectro radioeléctrico orientada al desarrollo de los servicios en mercados competitivos ayudan a una adecuada reasignación de recursos tendiente a maximizar el bienestar social.

Para fomentar la competencia entre redes hay que seguir introduciendo nuevas tecnologías, como las redes inalámbricas, el PLC (power line communication) y la televisión digital. Entre otros desafíos, habrá que: apresurar la introducción de nuevas concesiones, lo que implicará la oportuna dictación de normas técnicas y llamados a concurso, de ser necesario; simplificar la tramitación de concesiones a través de licencia única, y resolver de manera expedita recursos administrativos que intenten detener la entrada de nuevos actores.

\section{Igualdad en el acceso a Internet}

Es esencial aminorar las diferencias en el acceso a Internet —de carácter territorial, de género, étnico o etario- para asegurar un desarrollo no excluyente de la infraestructura de la información. Para lograrlo hay que evaluar las políticas de acceso a esta infraestructura, así como las políticas focalizadas de infraestructura para los sectores vulnerables y/o marginados. La infraestructura de la información ofrece a estos sectores un poderoso instrumento de acceso a mejores oportunidades y a una mejor calidad de vida. En este marco, la ruralidad y el aislamiento, la discapacidad o la pobreza urbana dejan de ser factores que limitan el desarrollo de las comunidades.

Entre los desafíos pendientes se encuentra el de avanzar en la construcción de una red nacional de infocentros que abarque las zonas rurales aisladas y las zonas urbanas de menores ingresos, y en la generación de instrumentos normativos orientados a promover la extensión de la cobertura de los servicios de telefonía a los hogares que aún no cuentan con ese servicio.
Ampliar de manera territorialmente equilibrada la capacidad y cobertura de la infraestructura de telecomunicaciones es clave para una integración no excluyente a través de Internet. Para fomentar la integración territorial es necesario evaluar la introducción de nuevos instrumentos que contribuyan a extender la infraestructura básica y de banda ancha a localidades extremas y, en particular, la red troncal de fibra óptica al extremo sur, completando así la red troncal de banda ancha continental.

\section{Eliminación de barreras al uso de Internet}

Hay que eliminar los factores que impiden un mayor uso de las nuevas tecnologías de información, inhibiendo las aplicaciones en la red, particularmente del comercio electrónico y demás actividades de negocios. Entre las tareas más urgentes en esta materia está la de seguir avanzando en la generación de instrumentos que fomenten y hagan más seguro el comercio a través de la red. Además, se debe fomentar la adecuación de la industria de distribución a los estándares y exigencias que se le imponen en países más desarrollados.

\section{Creciente presencia del Estado en las redes}

La incorporación del Estado en las redes constituye un elemento catalizador de la economía digital, que permite al mismo tiempo mejorar la calidad de vida de los ciudadanos y acercar los servicios públicos a sus usuarios. Si bien se ha progresado mucho en el diseño de programas orientados al gobierno en línea, es necesario concluir los proyectos en curso y educar a la población para que los utilice. Asimismo, el país tiene que seguir avanzando en el desarrollo de contenidos del sector privado.

\section{Fortalecimiento de la institucionalidad en el marco de la convergencia de medios}

Por la importancia atribuida a las telecomunicaciones y las tecnologías de la información en el desarrollo económico, es necesario adaptar la institucionalidad a la necesidad de absorber con rapidez las nuevas tecnologías y la información. Para ello, es preciso promover el desarrollo armónico de las regulaciones de los servicios convergentes y avanzar hacia la integración en un solo ministerio de los servicios de telecomunicaciones, tecnologías de la información, y ciencia y tecnología. Con esto se sustituiría la actual estructura transversal, que reduce la posibilidad de aprovechar las sinergias entre los sectores en convergencia y resta 
eficiencia a políticas derivadas de una institucionalidad focalizada en el desarrollo de la infraestructura sectorial. Un desafío pendiente es la creación de una superintendencia de telecomunicaciones que permita separar la función controladora de la función de fomento de políticas sectoriales.

\section{Participación en la construcción de la infraes- tructura}

Un elemento central en la construcción de la infraestructura de la información es la participación del sector privado, puesto que el sector de las telecomunica- ciones, al haber ido perdiendo cada vez más su carácter de servicio público tradicional, ${ }^{15}$ deberá avanzar hacia esquemas de autorregulación. En este ámbito, el sector público debe adoptar con mayor fuerza el papel de facilitador, contribuyendo a la expansión de las inversiones.

Finalmente, otro desafío es el de fortalecer la participación ciudadana, para utilizar Internet como herramienta de participación, estimular el desarrollo de contenido que contribuya a generar ciudadanos más informados, y potenciar el perfeccionamiento de los mercados a través del ejercicio de los derechos del ciudadano como consumidor.

\section{Bibliografía}

Cámara de Comercio de Santiago (2001): La economía digital en Chile, 2001, www.ccs.cl.

Cominetti, R. y E. Pérez (2001): Sector telecomunicaciones: modelos de institucionalidad alternativos, diciembre, inédito.

SUBTEL (Subsecretaría de Telecomunicaciones) (2000): Propuesta de un marco normativo para la introducción de la televisión digital terrestre en Chile, www.subtel.cl, Santiago de Chile, febrero.

(2001): Informe estadístico, $\mathrm{N}^{\circ} 3$, Santiago de Chile, diciembre.

(2002): Informe estadístico, $\mathrm{N}^{\circ}$ 4, Santiago de Chile, enero.

15 Véase un análisis de la naturaleza del servicio de telecomunicaciones en Cominetti y Pérez (2001). 